\section{PSicología IBEROAMERICANA}

Cevasco, Jazmín; Muller, Felipe

Conectividad Causal, Rol Conversacional y Confiabilidad del Hablante en el Recuerdo de Conversaciones

Psicología Iberoamericana, vol. 17, núm. 2, julio-diciembre, 2009, pp. 33-40

Universidad Iberoamericana, Ciudad de México

Distrito Federal, México

\section{Psicología lberoamericana}

ISSN: 1405-0943

psicología.iberoamericana@uia.mx

Universidad Iberoamericana, Ciudad de

México

México

Disponible en: http://www.redalyc.org/articulo.oa?id=133912609005

- Cómo citar el artículo

Número completo

- Más información del artículo

Página de la revista en redalyc.org

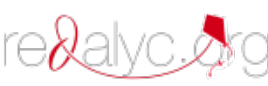

Sistema de Información Científica Red de Revistas Científicas de América Latina, el Caribe, España y Portugal Proyecto académico sin fines de lucro, desarrollado bajo la iniciativa de acceso abierto 


\title{
Conectividad Causal, Rol Conversacional y Confiabilidad del Hablante en el Recuerdo de Conversaciones
}

\author{
Causal Connectivity, Conversational Role, and Trustworthiness \\ of the Speaker in the Recall of Conversations
}

\author{
Jazmín Cevasco \\ Felipe Muller ${ }^{1}$ \\ Consejo Nacional de Investigaciones Científicas y Técnicas \\ Universidad de Buenos Aires- Universidad de Belgrano, Buenos Aires, Argentina
}

\section{RESUMEN}

Investigaciones acerca de la formación de la memoria grupal han destacado la importancia de los enunciados del narrador de un grupo y la confiabilidad de los hablantes. Investigaciones acerca de la comprensión del discurso han señalado la importancia del rol de las conexiones causales. El propósito de esta investigación fue explorar el rol de las conexiones causales de los enunciados, del rol conversacional y de la confiabilidad del hablante en el recuerdo de conversaciones grupales. Los resultados indicaron que tanto la conectividad causal, así como la confiabilidad del hablante tienen un efecto en la probabilidad de recuerdo de enunciados conversacionales, sugiriendo que una serie de factores psicológicos y sociales intervienen en la conformación de la memoria grupal.

Descriptores: Conectividad Causal, Rol Conversacional, Confiabilidad, Memoria Grupal.

\section{ABSTRACT}

Studies on the formation of group memory have highlighted the importance of the group narrator's statements, as well as the speaker's trustworthiness. Studies on the comprehension of discourse have underlined the importance of the role of causal connectivity. The purpose of this investigation was to explore the effect of the causal connections of a statement, the conversational role and the trustworthiness of the speaker in the recall of conversational statements. Results indicated that the number of causal connections and the trustworthiness of the speaker have an effect on the probability of recalling conversational statements, which in turn suggests that the formation of group memory involves a series of psychological and social factors.

Key words: Causal Connectivity, Conversational Role, Trustworthiness, Group Memory.

1 Para correspondencia: Jazmín Y. Cevasco, Instituto de Investigaciones, Facultad de Psicología, UBA, Independencia 3065, Piso $3^{\circ}$ Oficina 8 (1225) Buenos Aires, Tel: (0115411) 4772-7462, jazmincev@msn.com; Felipe Muller, Departamento de Investigaciones, Universidad de Belgrano, Zabala 1837, Piso 12 Of. 18 (1425) Buenos Aires (1426). Tel/Fax: (0054) 11-4788-5400, ext.2533 mullerf@fibertel.com.ar 


\section{EFECTO DE LA CONECTIVIDAD CAUSAL, EL ROL CONVERSACIONAL Y LA CONFIABILIDAD DEL HABLANTE EN EL RECUERDO DE CONVERSACIONES}

El desarrollo de memorias compartidas por un grupo de personas involucra una serie de factores psicológicos y sociales. En este estudio nos centraremos en el efecto de cuatro de ellos en el recuerdo de conversaciones grupales: el número de conexiones causales de los enunciados conversacionales, el rol conversacional del hablante, su confiabilidad y la calidad de las memorias de los participantes.

A pesar de la proliferación de estudios acerca de cómo el recuerdo de un mismo evento puede diferir de una persona a otra, sólo hasta fecha reciente se ha dedicado atención al estudio de cómo recuerdos diferentes de un evento pueden converger en una memoria compartida. El recuerdo así producido se define como memoria grupal, y es estudiado por la psicología de la memoria colectiva (Hirst \& Echterhoff, 2008; Hirst \& Manier, 2008).

Siguiendo a Wertsch (1998), la memoria grupal surge en parte debido a que los miembros de un grupo se apropian de lo que otros miembros ofrecen, $y$ en parte porque se resisten a su influencia. La apropiación puede definirse como el grado en que el recuerdo de una persona 'infecta' el recuerdo de otras. En otras palabras, la versión que un participante ofrece sobre un evento en una conversación grupal puede ser tomada por otros. Esta apropiación por imposición surge porque los participantes confunden el material que recibieron originalmente con lo que fue dicho en el grupo.

El diseño experimental estándar utilizado en las investigaciones sobre apropiación se basa en el trabajo de Sherif (1996). En la adaptación de su paradigma experimental, el experimentador pide a los participantes de un estudio que lean historias en forma individual y luego registra el recuerdo individual pre-grupal. En la segunda fase se lleva a cabo el recuerdo grupal, es decir, los participantes recuerdan juntos las historias leídas o escuchadas. En una tercera fase se les pide que vuelvan a recordar las historias en forma individual, registrando el recuerdo individual postgrupal. En la mayoría de los estudios que emplean este paradigma, el recuerdo grupal se lleva a cabo como una conversación entre los participantes. Durante esta conversación se establecen roles. Muchas veces uno de los participantes domina la discusión, pudiendo imponer su recuerdo o versión del pasado en los demás. Hirst y sus colegas se refieren a este participante como el Narrador (Hirst \& Manier, 1996), y lo definen como el que produce más del $25 \%$ de las unidades narrativas o enunciados conversacionales. Investigaciones previas han encontrado que cuanto más fuerte es el narrador en el recuerdo grupal (es decir, cuantos más enunciados conversacionales produce), más convergen los recuerdos individuales post-grupales en su versión. Cuando no hay un narrador en el grupo, el recuerdo postgrupal tiende a consistir en recuerdos compartidos previamente a la conversación (Cuc, Ozuru, Manier \& Hirst, 2006).

Como se menciono anteriormente, la resistencia es otro de los procesos a considerar. Los participantes de un grupo pueden también rechazar la versión del pasado brindada por otros. Una de las razones por las que puede suceder esto es porque desconfían de ellos. Quizás sucede porque creen que tienen motivos para mentir o desorientar, o que no son competentes en la tarea. En el área de psicología experimental, el efecto de la desconfianza suele ser explorado a través de experimentos en los que a los participantes se da una advertencia explícita acerca de la naturaleza desorientadora de la información dada por uno de los miembros del grupo (Loftus, 1979; Loftus, 1992; Loftus \& Hoffman, 1989). Estas advertencias suelen caracterizarlo como no confiable o incompetente en la tarea. Los resultados de estos estudios han permitido establecer que a partir de la advertencia, la capacidad de resistencia de los participantes aumenta (Greene, Flynn \& Loftus, 1982).

RESISTENCIA Y MEMORIA GRUPAL: INTERJUEGO ENTRE EL ROL CONVERSACIONAL, CONFIABILIDAD DEL HABLANTE Y CALIDAD DE LA MEMORIA ORIGINAL DEL PARTICIPANTE.

A fin de explorar el interjuego entre el rol conversacional, la confiabilidad del hablante y la calidad de la memoria original formada por el participante en el recuerdo de conversaciones, Muller \& Hirst (200) combinaron el paradigma de Sheriff (1996), con una técnica desarrollada por Bransford y Johnson (1973). 
Les pidieron a los participantes de su estudio que escucharan cuatro historias, y que las recordaran individualmente por escrito (recuerdo individual pregrupal). En una segunda fase, los participantes recordaron conjuntamente las historias escuchadas (recuerdo grupal). En una tercera fase, los participantes recordaron nuevamente de manera individual las historias por escrito (recuerdo individual postgrupal), y realizaron una prueba de reconocimiento. Utilizando la técnica de Bransford y Johnson (1973), los autores generaron cuatro versiones de las historias, cambiando seis detalles específicos en ellas (ítems críticos). La prueba de reconocimiento requirió que los participantes seleccionaran, de entre cuatro ítems posibles (correspondientes a las cuatro versiones de la historia) el que consideraran correcto. Por ejemplo, se les podían presentar 'los únicos sobrevivientes serán: 1) las cucarachas, 2) las palomas, 3) las arañas, 4) las ratas.' A fin de explorar el efecto de la desconfianza en el recuerdo de estos ítems, tres de los cuatro miembros de cada grupo recibieron una advertencia acerca de la naturaleza desorientadora de la información que daría el cuarto (se les dijo que había recibido una versión incorrecta de las historias). Siguiendo la técnica de Bransford y Johnson (1973), las historias fueron diseñadas de modo que resultaran difíciles de comprender sin estar acompañadas por un dibujo, y fueron presentadas de manera tal que siempre hubiese dos participantes que escucharan las historias con el dibujo, y dos sin él. Esta manipulación permitió comparar el efecto de haber formado una memoria de alta o baja calidad a partir de haber dispuesto o no del dibujo, en su capacidad de resistir a los ítems que dirían el narrador y el sujeto no confiable. Éstos podían coincidir en algunos casos. Es decir, el participante respecto del cual se daba la advertencia podía ser a su vez el que produjera la mayor cantidad de enunciados en el grupo. La inclusión de un ítem crítico dicho por el narrador o el sujeto no confiable en el recuerdo libre, y/o su selección en la prueba de reconocimiento fue tomada como evidencia de su apropiación por parte de los participantes.

En la tarea de recuerdo libre no se encontraron efectos de ninguna de las variables. El recuerdo de ítems críticos era muy escaso como para mostrar diferencias. En la prueba de reconocimiento se encontró que los participantes que habían escuchado la historia original sin el dibujo seleccionaban en mayor medida los ítems críticos producidos por el narrador y el sujeto no confiable, que los que habían escuchado la historia acompañada del dibujo. Es decir, los sujetos que poseían una versión más articulada y coherente eran más capaces de resistir a la influencia del narrador y el sujeto no confiable. A su vez, los participantes que habían escuchado la historia sin el dibujo seleccionaban más ítems críticos producidos por los no narradores confiables que por los no narradores no confiables, y seleccionaban más ítems críticos producidos por los narradores no confiables que por los narradores confiables. En otras palabras, la advertencia pareció permitir a los sujetos con una memoria de calidad baja monitorear los aspectos incorrectos del discurso de los sujetos no confiables no narradores, y resistir a su influencia, pero no logró disminuir la apropiación de ítems críticos dichos por los narradores no confiables. En el último caso, la advertencia parece haber enfocado la atención de estos sujetos en el monitoreo de los aspectos incorrectos del discurso del narrador, pero dado que su recuerdo de la historia demuestra ser más estructurado que el de ellos, terminan apropiándose de los ítems de su versión.

Dado que el rol conversacional, la confiabilidad del hablante y la calidad de la memoria original del oyente parecen jugar un rol en el recuerdo de ciertos ítems escuchados durante una conversación, resulta interesante continuar indagando qué otros ítems o enunciados incluyen los participantes en la representación que construyen del discurso escuchado. Esto es, aunque no se encontró un efecto del rol conversacional y la confiabilidad del hablante en el recuerdo de ítems críticos en los protocolos de recuerdo libre, aún queda por establecer si otros enunciados dichos por los narradores o sujetos no confiables sí fueron incluidos en estos protocolos.

\section{RECUERDO DE CONVERSACIONES $Y$ CONECTIVIDAD CAUSAL DE LOS ENUNCIADOS}

El recuerdo de conversaciones también se ha estudiado a partir del rol de las conexiones discursivas que los oyentes pueden establecer entre los enunciados. Dichos estudios acerca del rol de las conexiones discursivas causales en la comprensión del discurso escrito han mostrado que las oraciones con un gran número de 
causas o consecuencias son recordadas más frecuente (Trabasso \& van den Broek, 1985) y rápidamente (O’Brien \& Myers, 1987), y consideradas más importantes por los lectores (Trabasso \& Sperry, 1985) que las oraciones o enunciados con un menor número de conexiones causales. Considerando estos resultados, estos autores investigaron el procesamiento de las conexiones causales entre los enunciados en la comprensión de conversaciones. Para esto, presentaron un fragmento de un programa de radio a dos grupos de participantes. Al primero de ellos se le pidió que lo escuchara y que luego escribiera todo lo que recordaba de él y respondiera a una serie de preguntas. A un segundo grupo se le pidió que leyera la transcripción de la sección del programa y realizara las mismas tareas. La existencia de una conexión causal entre dos enunciados de los locutores se determinó a partir del Teoría de Red Causal (Trabasso \& van den Broek, 1985; Trabasso \& Sperry, 1985), cuyos criterios establecen que una causa descrita en un enunciado debe ser siempre temporalmente previa a la consecuencia descrita en otro enunciado. A su vez, la causa descrita en un enunciado debe estar activa $\mathrm{u}$ operante cuando ocurre la consecuencia. Además, la causa descrita en un enunciado debe ser necesaria para que se dé la consecuencia. Es decir, debe poder determinarse que si la causa descrita en un enunciado no hubiera ocurrido, la consecuencia descrita en otro enunciado tampoco hubiera sucedido. Los resultados indicaron que un enunciado era más recordado e incluido en respuestas a preguntas acerca del programa mientras mayor fuera su número de conexiones causales, independientemente del formato en que fuera presentado. A partir de este estudio, se puede proponer que el procesamiento de conexiones causales tiene un rol en la comprensión del discurso hablado por parte de oyentes que no participan de la conversación, y se puede plantear la necesidad de realizar más estudios para indagar acerca del procesamiento de conexiones causales por parte de oyentes que sean también hablantes en la conversación, a fin de determinar si el procesamiento de la causalidad jugaría el mismo rol, o si otras variables tendrían un papel más destacado.

\section{CONECTIVIDAD CAUSAL, ROL CONVERSACIONAL Y CONFIABILIDAD DEL HABLANTE}

Si se consideran los hallazgos en relación con el efecto del rol conversacional, la confiabilidad del hablante y la calidad de la memoria de los participantes de un grupo en el reconocimiento de ítems críticos (Muller \& Hirst, 2009), y se toma en cuenta la evidencia en favor del rol de la conectividad causal en el recuerdo de enunciados conversacionales por parte de oyentes que no participan de la conversación (Cevasco \& van den Broek, 2008), el propósito de este estudio fue reanalizar los datos de Muller y Hirst a partir del rol de las conexiones causales de los enunciados escuchados por los participantes en la historia originalmente presentada y en las conversaciones grupales en la tarea de recuerdo libre y su posible interacción con el rol conversacional del participante que produce el enunciado, su confiabilidad y la calidad de la memoria original del participante.

Dados los resultados de los estudios sobre la comprensión del discurso, esperamos que los enunciados que tuvieran un mayor número de conexiones causales en la historia original y en el recuerdo grupal fueran más recordados que aquellos que tuvieran un número menor de ellas, y por ende fueran más apropiados por los participantes que aquellos con un menor número de conexiones causales. A su vez, esperamos que los enunciados producidos por los narradores fueran más recordados que los producidos por los no narradores, dado que éstos son los participantes que hacen una mayor contribución a la conversación. También esperamos que los participantes que escucharan la historia original acompañada de un dibujo recordaran un mayor número de enunciados que los participantes que la escucharan sin él, dado que la disponibilidad del dibujo debería haberles permitido construir una memoria pre-grupal de mayor calidad. En cuanto a la confiabilidad del hablante, supusimos que los participantes que escucharan la historia original acompañada de un dibujo serían más capaces de resistir a la influencia de los sujetos no confiables, e incluirían menos enunciados dichos por ellos que los sujetos que escuchan la historia original sin el dibujo. 


\section{MÉTODO}

\section{Participantes}

Sesenta y ocho alumnos de la Universidad de Belgrano fueron reclutados a través de volantes pegados en las carteleras de la Universidad. Los participantes fueron divididos en 17 grupos de 4 alumnos. Los protocolos de recuerdo de los 17 narradores y los 17 sujetos no confiables fueron excluidos del análisis, dado que estos participantes sólo escuchan enunciados producidos por no narradores o por sujetos confiables. En algunos casos, el narrador y el sujeto no confiable coincidieron, por lo que el número final de participantes fue de 40 .

\section{Instrumentos}

A partir de las cuatro historias presentadas por Muller y Hirst (en evaluación), se seleccionó una de ellas para derivar una Representación de Red Causal de cada una de sus cuatro versiones (Tabla 1). La historia fue leída por un hablante nativo del español. Siguiendo los procedimientos propuestos por Trabasso y Sperry (1985), las cuatro versiones fueron segmentadas en enunciados. Los enunciados son unidades narrativas que contienen suficiente información para ser identificadas como causa o consecuencia de otro enunciado. Es decir, describen personas u objetos que sufren cambios como consecuencia de las acciones o procesos llevados a cabo por personas o mecanismos físicos. La existencia de una conexión causal entre dos enunciados fue determinada aplicando la siguiente prueba contrafáctica: siendo A y B dos enunciados, si el evento descrito en A no hubiera sucedido, entonces el evento descrito en B tampoco hubiera sucedido. Si se juzga que el contrafáctico es verdadero, se determina que $\mathrm{A}$ es causa de B. Por ejemplo, el enunciado (14) 'el automóvil será dañado' es causa de (16) 'el automóvil deberá ser removido posteriormente', dado que 'si el automóvil no fuera a ser dañado, 'no debería ser removido posteriormente'. A su vez, se derivó una red causal para cada una de las 17 transcripciones de las conversaciones grupales, siguiendo los mismos procedimientos. Es decir, se determinó la existencia de una conexión causal entre dos enunciados producidos por el mismo o diferentes participantes del recuerdo grupal

Tabla 1. Trascripción de una versión de la historia utilizada. (Los ítems críticos subrayados)

\begin{tabular}{|c|c|}
\hline Enunciados: & $\begin{array}{l}\text { Causalmente } \\
\text { conectado con }\end{array}$ \\
\hline 1. La coordinación del movimiento entre brazo izquierdo y brazo derecho, & $4-5$ \\
\hline 2. y entre pie izquierdo y pie derecho & $4-5$ \\
\hline 4. es necesaria para producir el efecto deseado. La fuerza del golpe & $1-2-5-6-7-13-14$ \\
\hline 5. depende del balanceo de la bola & $1-2-4$ \\
\hline 6. y el peso de la bola. & 4 \\
\hline 7. La fortaleza de las paredes debe ser también considerada en esta ecuación. & $4-8$ \\
\hline 8. Su estructura y su resistencia harían todo el emprendimiento más o menos duradero. & 7 \\
\hline 9. Quedarse sin nafta causaría la postergación de todo & \\
\hline 10. Pero el trabajo ha comenzado algún tiempo antes. & 11 \\
\hline 11. La gente y sus valijas fueron llevadas a otro lugar. & $10-12-13$ \\
\hline 12. Ahora, un galpón es el nuevo hogar. & 11 \\
\hline 13. Parece que las ratas van a ser las únicas víctimas. & $4-11$ \\
\hline 14. El automóvil será dañado, & $4-15-16$ \\
\hline 15. pero como fue abandonado, & $14-16$ \\
\hline 16. será otra cosa que ha de ser removida posteriormente. & $14-15$ \\
\hline
\end{tabular}


a partir de la aplicación del contrafáctico. Por ejemplo, un enunciado grupal (A) 'Hay que tener coordinación entre el pie y... la mano del hombre' se consideró causa de un enunciado grupal (B) 'para que la piedra pudiera golpear', dado que se puede proponer que 'si no hay coordinación entre el pie y la mano del hombre', 'la piedra no puede golpear'. A partir de la derivación de estas redes, se pudo establecer el número total de conexiones causales de cada enunciado escuchado en el grupo, considerando el número de conexiones que tenía en la versión de la historia original escuchada por el participante y el número de conexiones causales adicionales que se establecían durante el recuerdo grupal.

Los enunciados fueron a su vez analizados en relación con el rol conversacional (narrador-no narrador) y la confiabilidad (sujeto confiable- sujeto no confiable) del hablante que los producía. El rol conversacional se determinó con base en la cantidad de enunciados que el participante había aportado a la conversación. Siguiendo a Hirst y Manier (2008), se consideró narrador al participante que había producido la mayor cantidad de enunciados de entre los que habían producido más de un $25 \%$ de los enunciados en el recuerdo grupal, y no narradores al resto de los participantes.

\section{Procedimiento}

Se utilizó una adaptación del paradigma experimental de Sherif. En una primera sesión, cada participante escuchó individualmente cuatro historias (de las cuales una fue seleccionada) y luego las recordó de manera aislada, escribiendo todo lo que recordaba. Por cada grupo, dos de los participantes oyeron la historia con el dibujo, y dos sin él. En un segundo día, el participante que había producido la mayor cantidad de ítems clave en el recuerdo libre del primer día fue seleccionado como el participante no confiable del grupo. El investigador se reunió con los otros tres participantes que conformarían el grupo y les dijo que había dado accidentalmente una grabación equivocada de las historias al participante restante el día anterior, pero les pidió que no dijeran nada así podían seguir con el estudio de todas maneras. Luego, el experimentador pidió a los participantes recordar conjuntamente las historias, $\tan$ correcta y detalladamente como les fuera posible. El recuerdo grupal continuó hasta que los participantes estuvieron seguros de no recordar nada más.
La sesión grupal fue grabada. Al día siguiente, los participantes recordaron de nuevo las historias originales en forma individual. Se recordó a los tres participantes que habían recibido la advertencia acerca del cuarto participante, quien había recibido una historia equivocada, y que lo tuvieran en cuenta al recordar la historia original. Luego de que finalizaran la tarea de recuerdo libre, los participantes realizaron la tarea de reconocimiento.

\section{RESULTADOS}

Los protocolos de recuerdo individual post-grupal fueron segmentados en unidades narrativas. Las unidades narrativas tienen un sujeto y un predicado, y describen estados, acciones o eventos. Se consideró que un enunciado había sido recordado si el participante incluía toda la información o la idea central del mismo. Se realizó un ANOVA con conectividad causal (cero: 0 conexiones causales, baja: 1-3 conexiones causales, o alta: 4 o más conexiones causales), rol conversacional del hablante (narrador-no narrador), confiabilidad del hablante (confiable - no confiable) y calidad de la memoria original (alta: con dibujo- baja: sin dibujo) como variables independientes. La variable dependiente fue inclusión del enunciado en el protocolo del recuerdo individual post-grupal. Los resultados mostraron que el número de conexiones causales de un enunciado tenía un efecto en la probabilidad de que fuera recordado e incluido en estos protocolos, $F(2,219)=8.815$, $p<.001$. Los enunciados que tenían cero conexiones causales fueron menos recordados $(M=.1122, S D=$ .2934) que los enunciados que tenían un número bajo $(M=.2839, S D=.3017), t(149)=3.301, p=.001, y$ un número alto de conexiones causales $(M=.4676, S D=$ $.4128), t(118)=5.186, p<.001$. A su vez, los enunciados que tenían un número bajo de conexiones causales fueron menos recordados $(M=.2839, S D=.3017)$ que los enunciados con un alto número de conexiones causales $(M=.4676, S D=.4128), t(171)=3.382, p=.001$. La variable rol conversacional no tuvo un efecto, $F$ ( 1 , $220)=.025, p=.825$. El contexto desde el cual el participante escuchaba la historia original también tuvo un efecto, $F(1,220)=5.018, p=.026$. Los sujetos que escucharon las historias originales acompañadas de un dibujo recordaron más enunciados $(M=.4661, S D=$ .3864) que aquellos que escucharon la historia original 
sin el dibujo $(M=.2350, S D=.3287)$. La confiabilidad del hablante tuvo un efecto a su vez, $F(1,220)=30.058$, $p<.001$. Los enunciados producidos por hablantes confiables fueron más recordados $(M=.3243, S D=$ $.3810)$ que los enunciados producidos por hablantes no confiables $(M=.2802, S D=.3377)$. La única interacción significativa encontrada fue entre confiabilidad y narrador $F(1,218)=6.978, p=.009$. Los enunciados producidos por los narradores confiables fueron más recordados $(M=.3504, S D=.3627)$ que los enunciados producidos por narradores no confiables $(M=.1626$, $S D=.2547), t(97)=2.765, p=.007, y$ no narradores no confiables $(M=.0575, S D=.1538), t(96)=4.601, p<$ .001. A su vez, los enunciados producidos por no narradores confiables fueron más recordados $(M=.4350$, $S D=.3926)$ que los producidos por narradores no confiables $(M=.1626, S D=.2547), t(122)=3.882, p<.001$, y no narradores no confiables $(M=.0575, S D=.1538)$, $t(121)=5.583, p<.001$.

\section{DISCUSIÓN}

Este estudio investigó el efecto del rol conversacional, la confiabilidad del hablante, la calidad de la memoria original y la conectividad causal de los enunciados en el recuerdo de conversaciones en un contexto grupal.

A partir de investigaciones acerca del rol conversacional en la memoria grupal, se esperó que el narrador de un grupo fuera más capaz de imponer sus enunciados en el recuerdo de otros participantes que los no narradores, ya que es el participante que hace una mayor contribución a la conversación. A su vez, se confió en que la formación de una memoria de alta calidad basada en la presencia del dibujo permitiera al participante recordar más enunciados y ser más capaz de resistir a la influencia de los enunciados del narrador y el sujeto no confiable que la posesión de una memoria de baja calidad. A partir de investigaciones acerca del rol de la conectividad causal en la comprensión de discurso, se supuso que aquellos enunciados que tuvieran más conexiones causales con otros fueran más recordados que aquellos que tuvieran menos conexiones causales, y permitieran a su vez al participante resistir a la influencia del narrador y el sujeto no confiable, coincidieran éstos o no.

Los resultados indicaron un efecto para la conectividad causal de los enunciados, para la confiabilidad del hablante que produce el enunciado, y para la calidad de la memoria original del participante. A su vez, indicaron una interacción entre la confiabilidad del hablante y su rol conversacional. Es decir, la inclusión de un enunciado en el recuerdo que un participante construye con respecto a una conversación de la que participó parece darse a partir de su capacidad de relacionarlo con otros enunciados, de cuán articulada y coherente es la memoria que construyó del material, $y$ de que no haya existido una advertencia acerca del hablante que lo produjo. Por otra parte, la influencia que puede tener un narrador parece depender de su confiabilidad. Un narrador confiable es más capaz de imponer enunciados que un narrador no confiable. $Y$ a su vez, un no narrador confiable es más capaz de imponer enunciados que uno no confiable.

Considerando los resultados de este estudio y los del de Muller y Hirst (en evaluación), se puede proponer que los participantes de una conversación son más capaces de resistir a la influencia del narrador y del sujeto no confiable cuando se les pide recordar la representación global que construyeron de la conversación, que cuando se les requiere recordar ítems específicos escuchados. Esto puede deberse a que, cuando deben recordar la conversación como un todo, pueden apoyarse en las conexiones causales que establecieron entre los enunciados, y apropiarse de los que tienen más conexiones, independientemente del rol conversacional o de la confiabilidad del hablante que los produce, resistiendo a la influencia del narrador y el sujeto no confiable. En el caso del recuerdo de ítems específicos, la influencia de los narradores confiables o no confiables parece hacerse más fuerte, ya que como demuestran poseer una representación coherente y articulada del material, logran imponer los ítems de su versión a los participantes que no cuentan con ella. 


\section{REFERENCIAS}

Bransford, J.D. \& Johnson, M.K. (1973). Considerations of some problems of comprehension. En W. G. Chase (Ed.). Visual information processing. (pp. 383-462). New York: Academic Press.

Cevasco, J. \& van den Broek, P. (2008). The importance of causal connections in the comprehension of spontaneous discourse. Psicothema, 20, 801-806.

Cuc, A., Ozuru, Y., Manier, D., \& Hirst, W. (2006). On the formation of collective memories: The role of a dominant narrator. Memory and Cognition, 34, 752762.

Diehl, M. \& Stroebe, W. (1991). Productivity loss in idea-generating groups: Tracking down the blocking effect. Journal of Personality and Social Psychology, 61, 392-403.

Greña, E., Flynn, M. \& Loftus, E. (1982). Inducing resistance to misleading information. Journal of Verbal Learning and Verbal Behavior, 21, 207-219.

Hirst, W. \& Echterhoff, G. (2008). Creating shared memories in conversation: toward a psychology of collective memory. Social Research, 75, 1-34.

Hirst, W. \& Manier, D. (1996). Social influences on remembering. En D. Rubin (Ed.), Remembering the past. New York: Cambridge University Press.

Hirst, W., \& Manier, D. (2008). Towards a psychology of collective memory. Memory, 16, 183-200.

Loftus, E. F. (1979). Eyewitness testimony. Cambridge, MA: Harvard University Press.

Loftus, E. F. (1992). When a lie becomes a memory's truth: Memory distortion after Resistance to the in- fluences of others: limits to the formation of a collective memory through conversational remembering.

Loftus, E.F., Hoffman, H.G. (1989). Misinformation and memory: The creation of new memories. Journal of Experimental Psychology: General, 118, 100-104.

Manier, D. \& Hirst, W. (2009). A cognitive taxonomy of collective memories. En A. Nunning \& A. Eril (Eds). Cultural memory studies: An International and interdisciplinary handbook. Berlin: de Gruyter.

Muller, F., \& Hirst, W. (2009). Resistance to the influences of others: limits to the formation of a collective memory through conversational remembering. Applied Cognitive Psychology, 23, 9, 1426.

O’Brien, E. J. \& Myers, J. L. (1987). The role of causal connections in the retrieval of text. Memory \& Cognition, 15, 419-427.

Sherif, M. (1996). The psychology of social norms. New York: Harper-Row.

Trabasso, T. \& Sperry, L. L. (1985). Causal relatedness and importance of story events. Journal of Memory and Language, 24, 595-611.

Trabasso, T. \& van den Broek, P. (1985). Causal thinking and the representation of narrative events. Journal of Memory and Language, 24, 612-630.

Wertsch, J. (1998). Mind in action. NY: Oxford University Press.

Wilkes-Gibbs, D. \& Kim, P. (1991). Discourse influences on memory for visual forms. Paper presented to the 1991 meeting for the Psychonomic Society, SF. 